\title{
Nonlinear Aerodynamic Modeling using \\ Multivariate Orthogonal Functions
}

by

Eugene A. Morelli

Lockheod Englneerlng and Sciences Co.

Alrcraft Guldance and Control Branch

NASA Langley Research Center

March 19, 1993

Complete detalis can be found in:

Morelli, E.A., Nonlinear Aerodynamlc Modeling using

Multivariate Orthogonal Functions, AIAA paper 93-3636,

Atmospheric Flight Mochanics Conference,

Montorey, CA, August 1993.

The problem to be addressed in this work is that of modeling nondimensional force and moment aerodynamic coefficents over the entire subsonic envelope. The particular application discussed here is the $Z$ force coefficient for the F-18 High Angle of Attack Research Vehicle (HARV).

PAECEDING PAGE ELAINK NOT FLMED 


\section{System Identification for Aircraft}

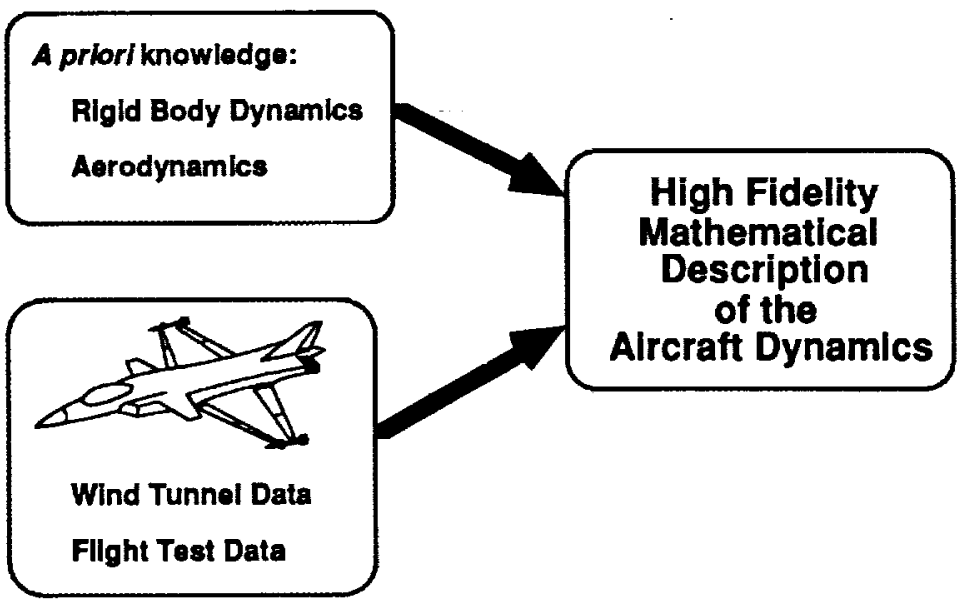

In general for high fidelity math models of aircraft dynamics, some a priori knowledge is combined with experimental data. The experimental data generally supplies the information on the aerodynamics particular to the aircraft under study. 


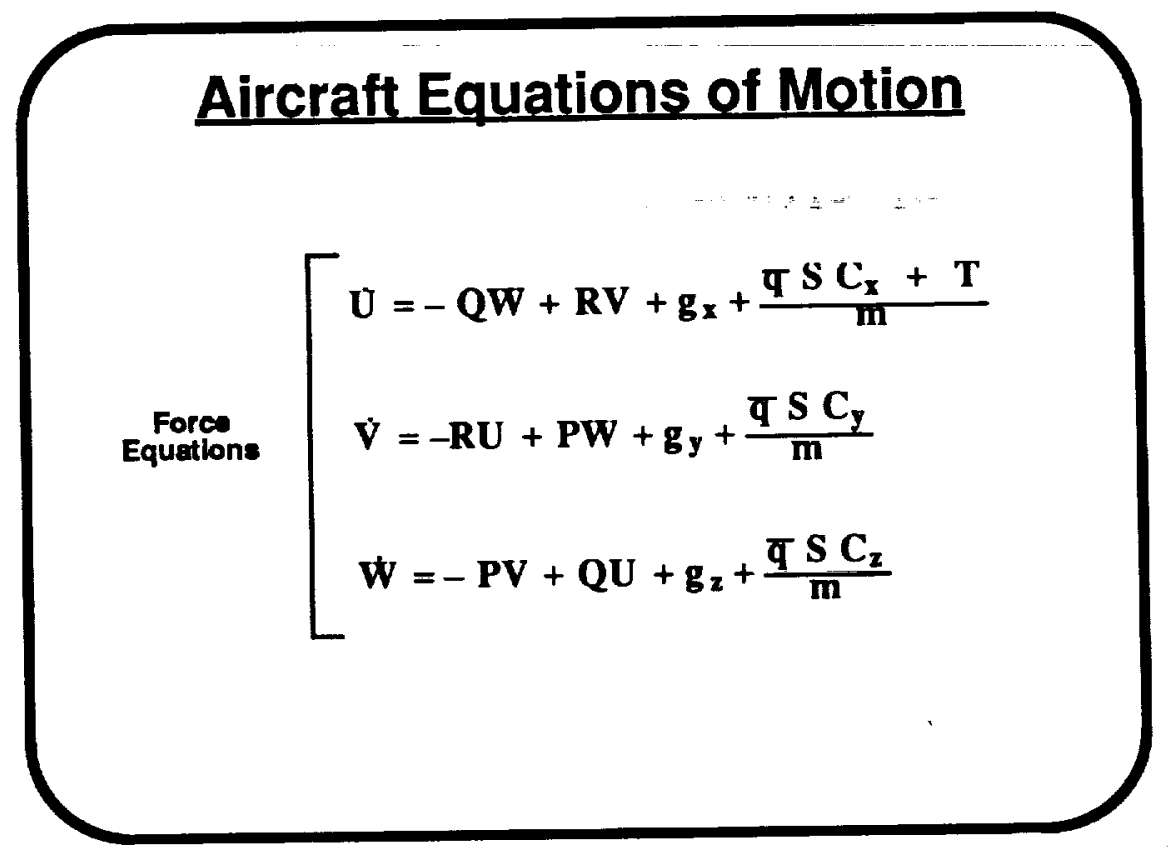

Shown here are the three nonlinear equations of motion for translational motion of an aircraft. Moment equations are omitted for simplicity, but the same discussion can be applied to them as well. The objective is to find a model for the aerodynamic coefficients, like $\mathbf{C z}$, in terms of the aircraft states and outputs and their derivatives, and the controls. This would allow prediction of dynamic behavior when such models are substituted into the equations above, along with a model for the thrust. 


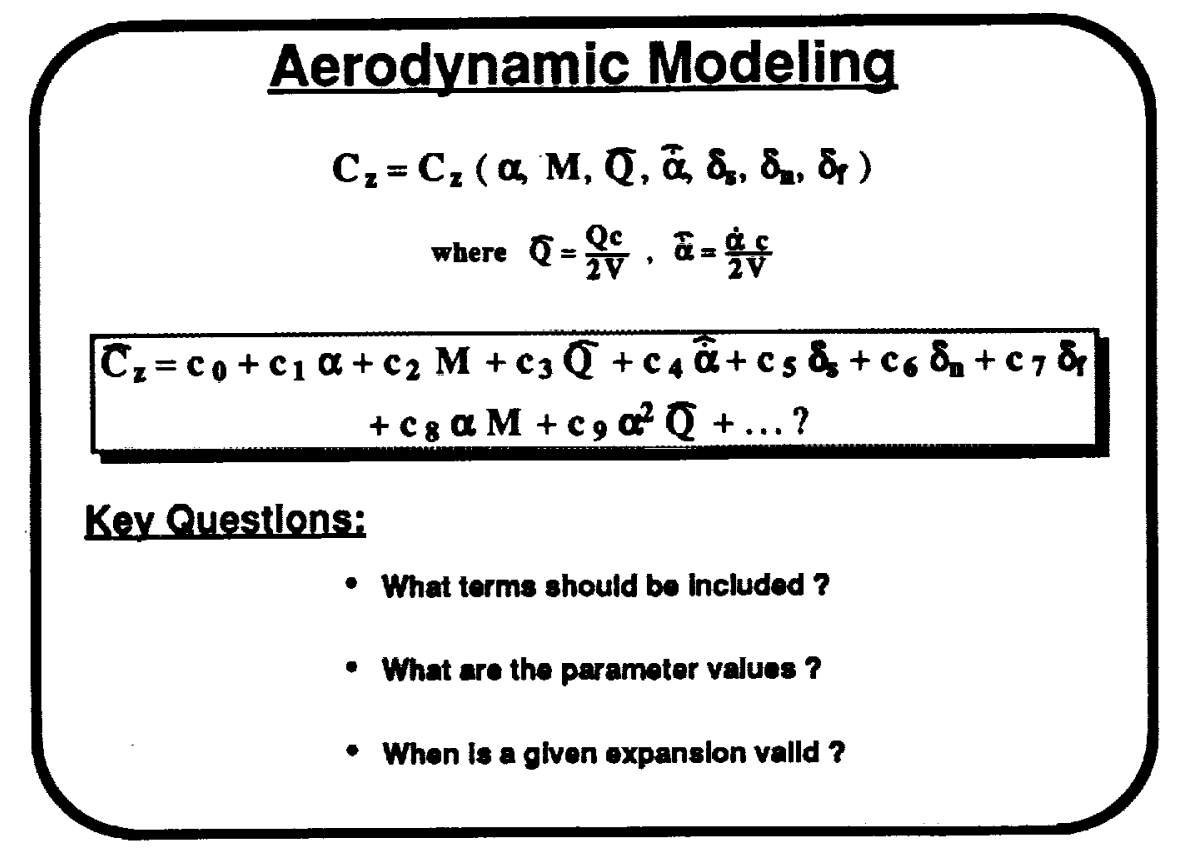

Part of the a priori knowledge is the functional dependence shown on the first line of this slide. Using this information, a polynomial model is postulated which is analogous to a truncated multivariable Maclaurin series expansion for $\mathbf{C z}$. This approach gives rise to the three questions posed at the bottom of the slide. 
Aerodynamic Coefficient Expansion

$$
\begin{gathered}
C_{2}=c_{0}+c_{1} \alpha+c_{2} M+c_{3} \bar{Q}+c_{4} \hat{\alpha}+c_{5} \delta_{5}+c_{6} \delta_{n}+c_{7} \delta_{7} \\
+c_{8} \alpha M+c_{9} \alpha^{2} \bar{Q}
\end{gathered}
$$

Usually difficult to accurately estimate parameters in this expansion from experimental data because:

- Experimental data does not contaln sufficient information

- Control system or the experiment correlates the regressors

- The model structure is in question

Assuming now that the number of terms to be included has been determined somehow (perhaps using stepwise regression), and the range of validity of this expansion has also been determined, there remain further difficulties in accurately estimating the parameter values (the c's) in the model. These difficulties are listed on this slide. 
Aerodynamic Coefficient Expansion in Terms of Orthogonal Functions

$$
C_{z}=a_{1} \xi_{1}+a_{2} \xi_{2}+\ldots+a_{n} \xi_{n}
$$

where In general

- $\xi_{i}=\xi_{1}\left(\alpha, M, \widehat{Q}, \hat{\alpha}, \delta_{s}, \delta_{n}, \delta_{1}\right) \quad$ for $i=1,2, \ldots, n$

- $\xi^{\mathrm{T}} \xi_{\mathrm{j}}=\mathbf{0}$ for $\mathbf{i} \neq \mathbf{j}$

- $\mathbf{a}_{1}=\frac{\xi_{1}^{\mathrm{T}} C_{2}}{\xi_{1}^{\mathrm{T}} \xi_{1}} \rightarrow \begin{aligned} & \text { the } 1^{\text {th }} \text { term in the model } \\ & \text { depends only on } C_{2} \text { and } \xi_{1}\end{aligned}$

If instead of ordinary polynomials in the expansion for $\mathrm{Cz}$, orthogonal polynomials with the properties shown on this slide were used, the difficulties in accurately estimating the model parameters disappear. This is because the computation of the value of the model parameter for term i depends only on the measured data $(\mathrm{Cz})$ and orthogonal function $\mathbf{i}$. This follows from the expansion for $\mathrm{Cz}$ in terms of orthogonal polynomials and the properties of orthogonal polynomials. 


\section{Model Structure Determination}

- Minimum predlcted square error ls the criterion for whlch orthogonal functions should be Included in the model :

$$
\text { PSE }=\frac{1}{N} \sum_{i=1}^{N}\left(C_{z_{1}}-C_{z i}\right)^{2}+2 \sigma_{p}^{2} \frac{n}{N}
$$

where

$$
\begin{gathered}
\sigma z=\frac{1}{N} \sum_{i=1}^{N}\left(C_{z_{1}}-C_{z}\right)^{2} ; \quad C_{z}=\frac{1}{N} \sum_{1=1}^{N} C_{z_{1}} \\
\sigma_{p}^{2}=\frac{\sigma_{0}^{2}}{2}
\end{gathered}
$$

The orthogonal functions to be included in the model can be determined in a straightforward way using the predicted square error (PSE) criterion, where $\boldsymbol{n}$ is the number of terms in the model and $\mathrm{N}$ is the number of data points. Since each term in the orthogonal function expansion is independent of all the others, the decision of whether or not to include each individual orthogonal function term can be made based on whether or not its inclusion reduces PSE. This can be done for each term sequentially and without regard to any other terms already in the model. 


\section{Finding Ordinary Polynomial Models}

$$
C_{2}=a_{1} \xi_{1}+a_{2} \xi_{2}+\ldots+a_{n} \xi_{n}
$$

It is possible to:

- Generate orthogonal functions based on the data using a technique similar to Gram-Schmidt orthogonalbatlon

- Determine the orthogonal function expansion by decrease in flt error ve. additional terms

- Decompose each orthogonal function without ambigulty in torms of ordinary polynomlals, e.g.

$$
\xi_{1}=b_{1} \alpha+b_{2} M+b_{3} \alpha \delta_{s}
$$

- Arrive at an ordinary polynomlal model with adequate structure and accurately estimated parameters

Details of how to generate multivariate orthogonal functions based on the data can be found in the reference given on the first slide. In that reference, it is also shown that each orthogonal function can be decomposed precisely into an expansion in terms of ordinary polynomials. Once the model structure determination is done in terms of orthogonal functions, the expansions for each included orthogonal function in terms of ordinary polynomials is substituted and common terms are combined, resulting finally in an ordinary polynomial model with adequate model structure and accurately estimated model parameters 


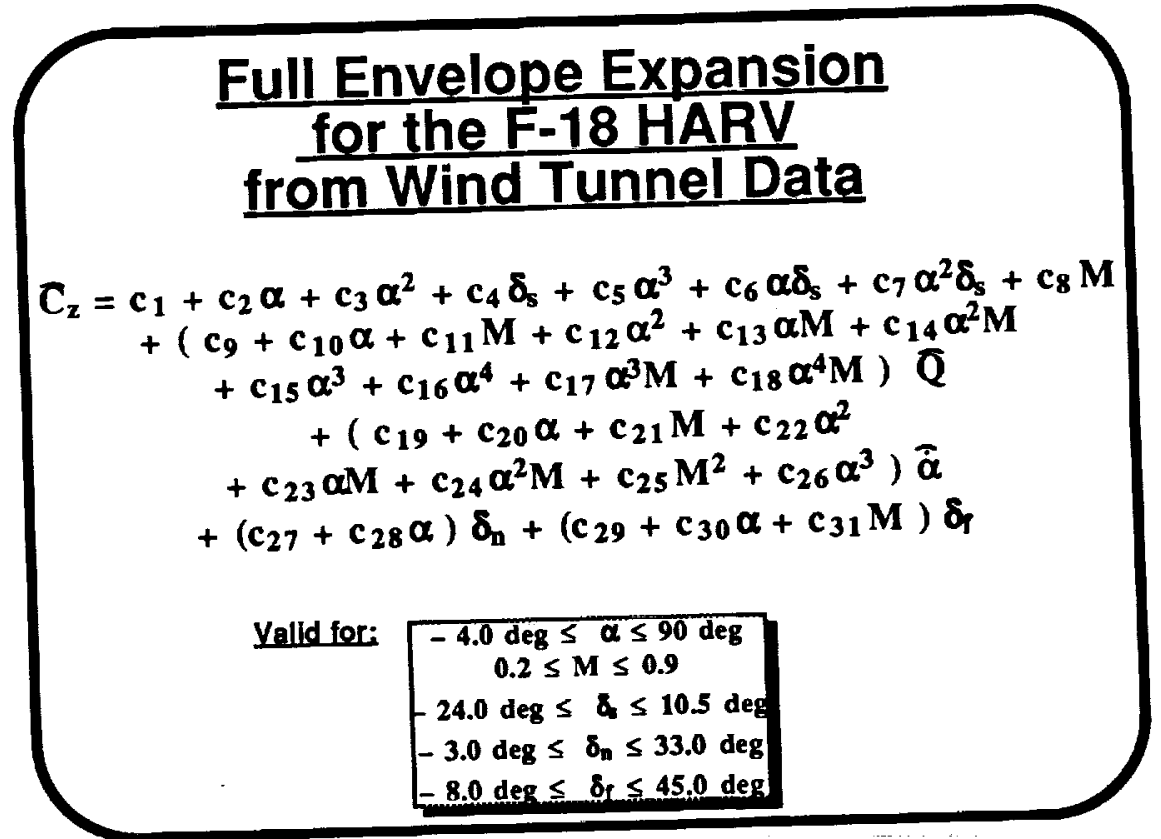

This is the result of using the technique developed here to model the $Z$ aerodynamic force coefficient for the F-18 HARV over the entire subsonic flight envelope, based on a wind tunnel database. 


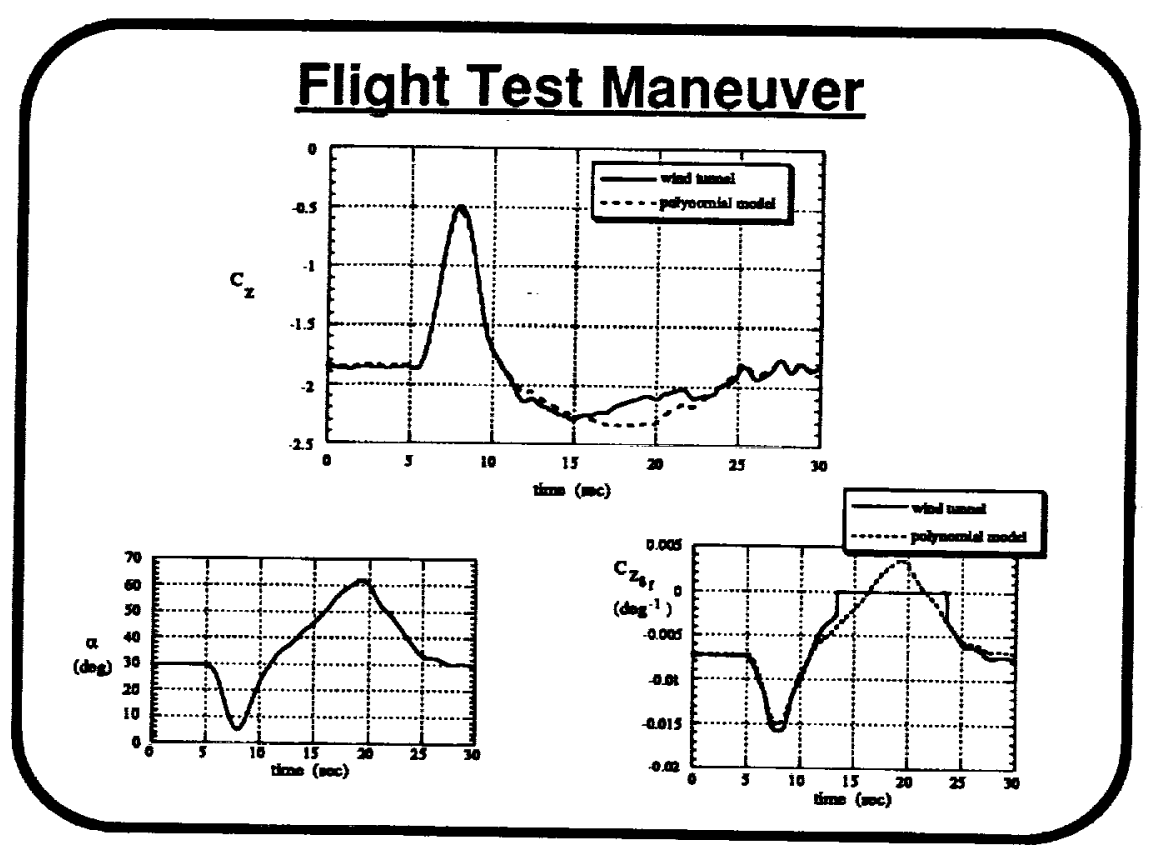

A prediction case for the polynomial model is shown here, using independent variable time histories taken from flight data. The prediction is excellent, with slight error in the time period from 13 to 23 seconds. This was traced to the fact that the wind tunnel database zeroed certain components of the $Z$ force coefficient when angle of attack exceeded 40 degrees, because of lack of data. The polynomial model, on the other hand, extrapolated reasonably, as shown on the lower right plot. 


\title{
Features of the Model
}

- Large tables of aerodynamlc data reduced to a small number of paramotors

- Smooth eorodynamic functlons and partial derlvatives

- Better Insight on the aerodynamle functional dependence

- Potential for automated simulator updates

- One model for the entire flight envelope

\begin{abstract}
Along with the improved accuracy of model parameters and excellent prediction capability inherent in the modeling approach discussed here, this slide outlines the some features of nonlinear aerodynamic models generated with this technique.
\end{abstract}




\section{Conclusion}

Accomplishment

- Developed and demonstrated a full envelope nonlinear aerodynamlc modeling technlque

Applications

- Flight Simulators

- Global Stability and Control Analysis

- Dynamic Analysis

- High Angle of Attack Control Design

The fact that the aerodynamic model for a full subsonic envelope can be made compact and smoothly differentiable while retaining excellent predictive capability has potential utility in a wide range of activities. 